\title{
Programmed Death Ligand 1 Expression and Tumor Infiltrating Lymphocytes in Neurofibromatosis Type 1 and 2 Associated Tumors
}

\author{
Shiyang Wang ${ }^{1}$, Benjamin Liechty ${ }^{2}$, Seema Patel ${ }^{1}$, Jeffrey S. Weber ${ }^{3}$, Travis J. Hollmann ${ }^{4}$, \\ Matija Snuderl $^{2}$, and Matthias A. Karajannis ${ }^{5}$ \\ ${ }^{1}$ Department of Pediatrics, Division of Pediatric Hematology/Oncology, NYU Langone Health, \\ New York, NY \\ ${ }^{2}$ Department of Pathology, Division of Neuropathology, NYU Langone Health, New York, NY \\ ${ }^{3}$ Laura and Isaac Perlmutter Cancer Center, NYU Langone Health, New York, NY \\ ${ }^{4}$ Department of Pathology, Memorial Sloan Kettering Cancer Center, New York, NY \\ ${ }^{5}$ Department of Pediatrics, Memorial Sloan Kettering Cancer Center, New York, NY
}

\begin{abstract}
Introduction-Immune checkpoint inhibitors targeting programmed cell death 1 (PD-1) or its ligand (PD-L1) have been shown to be effective in treating patients with a variety of cancers. Biomarker studies have found positive associations between clinical response rates and PD-L1 expression on tumor cells, as well as the presence of tumor infiltrating lymphocytes (TILs). It is currently unknown whether tumors associated with neurofibromatosis types 1 and 2 (NF1 and NF2) express PD-L1.
\end{abstract}

Methods-We performed immunohistochemistry for PD-L1 (clones SP142 and E1L3N), CD3, CD20, CD8, and CD68 in NF1-related tumors (10 dermal and 6 plexiform neurofibromas) and NF2-related tumors (10 meningiomas and 10 schwannomas) using archival formalin-fixed paraffin-embedded tissues. Expression of PD-L1 was considered positive in cases with $>5 \%$ membranous staining of tumor cells, in accordance with previously published biomarker studies.

Results-PD-L1 expression in tumor cells (using the SP142 and E1L3N clones, respectively) was assessed as positive in plexiform neurofibromas (6/6 and 5/6) dermal neurofibromas (8/10 and 6/10), schwannomas (7/10 and 10/10), and meningiomas (4/10 and 2/10). Sparse to moderate presence of CD68, CD3, or CD8 positive TILs was found in 36 (100\%) of tumor specimens.

Conclusions-Our findings indicate that adaptive resistance to cell-mediated immunity may play a major role in the tumor immune microenvironment of NF1 and NF2-associated tumors. Expression of PD-L1 on tumor cells and the presence of TILs suggest that these tumors might be

Correspondence: Matthias A. Karajannis, MD, MS, Department of Pediatrics, Memorial Sloan Kettering Cancer Center, 1275 York Ave, Box 234, New York, NY 10065, Tel: (212) 639-3171, Fax: (212) 717-3239, karajanm@ mskcc.org.

Conflict of Interest: The authors declare that they have no conflict of interest. 
responsive to immunotherapy with immune checkpoint inhibitors, which should be explored in clinical trials for NF patients.

\section{Keywords}

neurofibromatosis type 1; neurofibromatosis type 2; neurofibroma; schwannoma; meningioma; tumor infiltrating lymphocytes; programmed death-ligand 1

\section{Introduction}

Neurofibromatosis type 1 (NF1) and type 2 (NF2) represent two distinct, autosomal dominant tumor predisposition syndromes caused by germline mutations in the $N F 1$ and $N F 2$ gene respectively. The most common tumor types arising in NF1 patients are cutaneous neurofibromas and plexiform neurofibromas. The predominant tumor types arising in patients with NF2 are multiple schwannomas, most commonly bilateral vestibular schwannomas, and meningiomas. While the majority of NF-related tumors are histologically benign, they are a leading cause of morbidity and mortality in NF patients. Standard therapies have traditionally included surgery and radiation therapy, and molecular targeted therapies have recently emerged with some success in subsets of NF-related tumors [1-4]. Despite these advances, there remains an urgent need for novel, more effective and less toxic therapies for NF patients, especially those with large tumor burden.

Immune checkpoint inhibitors, including antibodies targeting programmed death ligand-1 (PD-L1) and its receptor, PD-1 have been emerged as a promising therapeutic approach for a variety of cancers. As members of the immune checkpoint family, PD-L1 and PD-1 function as physiological immune suppressors to limit $\mathrm{T}$ cell response and help avoid autoimmunity [5]. PD-L1 overexpression is found in various tumor types and associated with poor prognosis [6]. Activation of cytokines and/or oncogenic signaling pathways, including EGFR tyrosine kinase signaling, MAP kinase and PI3/Akt-mTOR pathways, may lead to upregulation of PD-L1 [6-7]. The engagement of PD-1 on T cells with PD-L1on tumor cells leads to an inactive T-cell status with decreased T cell proliferation and survival, as well as increased suppressor $\mathrm{T}$ cell differentiation [8]. Therefore, blockage of the immune checkpoint PD L-1/PD-1axis may reactivate CD8 positive cytotoxic $\mathrm{T}$ cells that recognize and eliminate tumor cells. The FDA has approved anti-PD1 and anti-PD-L1 antibodies to treat a growing spectrum of cancers, including renal cell, colon and rectum, bladder, head and neck, Merkel cell, and non-small cell lung cancer, as well as Hodgkin's lymphoma and melanoma [6]. Many clinical trials are currently underway to investigate the therapeutic potential of these antibodies in various tumors, alone or in combination with other therapies [8-9].

NF1 and NF2 associated tumors are characterized as "inflammatory" tumors with abundant tumor macrophage infiltration, elevated serum IFN $\gamma$ levels, and dysregulation of oncogenic signaling pathways [10-13]. These elevated cytokines and amplified signaling pathways may provide a potential mechanism for upregulation of PD-L1 expression in tumor cells $[1,3$, 14]. Therefore, we hypothesized that PD-1/PD-L1 axis-mediated immune escape mechanisms might also exist in NF related tumors. 
It has been recognized that tumor cell membrane expression of PD-L1, as well as tumor infiltrating CD8 positive cytotoxic $\mathrm{T}$ cells represent predictive biomarkers associated with therapeutic response to PD-1 blockade [15]. We hypothesized that adaptive resistance to cell-mediated immunity may play a major role in the tumor immune microenvironment of NF1 and NF2-associated tumors, which would suggest that immune checkpoint inhibitors might be a new therapeutic strategy for the treatment of NF-associated tumors. Therefore, we examined PD-L1 membrane expression in NF1-associated cutaneous neurofibromas and plexiform neurofibromas as well as NF2-associated meningiomas and schwannomas by immunohistochemical staining. We further characterized the immune environment in these tumors by assessing the infiltration of lymphocytes, including CD8 positive cytotoxic $\mathrm{T}$ cells.

\section{Methods}

\section{Patients and Tissue Specimens}

This study was initiated under an Institutional Review Board (IRB) approved protocol at New York University-Langone Health. For this study, we selected archival formalin-fixed paraffin-embedded (FFPE) tumor samples from patients who met clinical diagnostic criteria for NF1 or NF2, excluding patients who had previously been treated with chemotherapy or radiation therapy. In total, 16 NF1 related tumors (10 cutaneous neurofibromas and 6 plexiform neurofibromas) and 20 NF2-related tumors (10 meningiomas and 10 schwannomas) with adequate quality and quantity of tissue were selected for further study, after review of slides by two neuropathologists (MS and BL).

\section{Immunohistochemistry}

Immunohistochemistry (IHC) was performed at NYU Langone Health on 5- $\mu$ m FFPE human tissue sections using the following unconjugated antibodies: rabbit anti-human CD3, human CD8, clone SP57 (Ventana Medical Systems. Cat\# 790-4460 Lot\# C05708 RRID: AB_2335985274), mouse anti-human CD20, clone L26 (Ventana Medical Systems. Cat\# 760-2531 Lot\# C06261 RRID: AB_2335956), mouse anti-human CD68, clone Kp1 (Ventana Medical Systems. Cat\# 790-2931 Lot\# B09466 RRID: AB_2335972), and CD274, Programmed Cell Death 1 Ligand 1, clone SP142 (Spring Biosciences Cat\# M4420 Lot\# 141027LVK RRID: unassigned). Chromogenic IHC was performed on a Ventana Medical Systems Discovery XT instrument with online deparaffinization and using Ventana's reagents and detection kits, unless otherwise noted. With the exception of CD20, markers were antigen retrieved in Ventana Cell Conditioner 1 (Tris-Borate-EDTA) as follows: CD68; 20 minutes, CD3 and CD8; 36 minutes, and CD274; 52 minutes. Endogenous peroxidase activity was blocked with $3 \%$ hydrogen peroxide for 4 minutes. All antibodies were applied neat and incubated for 30 minutes except for CD274 which was diluted 1:50 in Dulbecco's phosphate buffered saline (Invitrogen Life Technologies) and incubated for 60 minutes at room temperature. CD3 and CD8 were detected with goat anti-rabbit and CD20 and CD68 were detected goat anti-mouse, multimers incubated for 8 minutes respectively. The immune complexes were visualized with 3,3 diaminobenzidene and enhanced with copper sulfate. PDL1 was detected with hapten linked, anti-rabbit multimer incubated for 32 minutes followed by anti-hapten horseradish peroxidase conjugate for 32 minutes. This was followed 
by hapten-conjugated tyramide and horseradish peroxidase incubated for 32 minutes. The complex was visualized with 3,3 diaminobenzidene and enhanced with copper sulfate. Slides were washed in distilled water, counterstained with hematoxylin, dehydrated and mounted with permanent media.

Immunohistochemical staining using an additional PD-L1 antibody (Cell Signaling Technology, clone E1L3N) was performed was performed at Memorial Sloan Kettering Cancer on formalin-fixed paraffin-embedded archival tissue blocks sectioned onto charged (plus) slides at $4 \mu \mathrm{m}$ and run on a Leica Bond RX automated staining machine at a concentration of $2.02 \mu \mathrm{g} / \mathrm{ml}$ following antigen retrieval in ER2 for 30 minutes at room temperature with primary antibody incubation for 30 minutes and developed using a Leica Bond polymer refine IHC detection system. PDL1 (Cell Signaling Technology; clone E1L3N) staining was run on a Leica Bond RX automated staining machine at a concentration of $2.02 \mu \mathrm{g} / \mathrm{ml}$ following antigen retrieval in ER2 for 30 minutes at room temperature with primary antibody incubation for 30 minutes and developed using a Leica Bond polymer refine IHC detection system.

Expression of PD-L1 and immune cell markers was evaluated blindly by two neuropathologists. The scores of PD-L1 staining were counted based on the amount of cell membrane expression on tumor cells versus total tumor cells in the tissue sections. PD-L1 positive expression was defined as $>5 \%$ tumor cells with cell membrane staining. The density of CD8+, CD3+, CD20+ and CD68+ immune cells was assessed based on overall impression of sparse, moderate, or intense at low microscopic magnification (100x). Staining was assessed considering the morphology of inflammatory cells. CD8+, CD3+, $\mathrm{CD} 20+$ cells were small with high nuclear/cytoplasmic ration compatible with lymphocytes. For CD68+, only cells with abundant clear/foamy cytoplasm consistent with macrophages were considered. All IHC sections were compared to matching H\&E sections to ensure that expression of inflammatory cell markers is not present in tumor cells. In addition, staining was assessed as patchy when there was marked variability in expression across the entire slide. Then, cell density was further assessed by cell counting within the most densely infiltrated focus arising within the tumor tissue identified in a single high power (400x) field, taking care to exclude immunopositive cells not arising in a background of tumor (e.g. in perivascular spaces or regions of hemorrhage) or regions of spurious expression (e.g. artifactual staining, or expression in cells with equivocal morphologic features).

\section{Statistical Analysis}

The chi-square test was utilized to test group association between PD-L1 expression and CD8 positive T cell density. A significance $p$ value of 0.05 was applied. Statistics were calculated using freely available software at www.socscistatistics.com.

\section{Results}

\section{Patient Characteristics}

The patients' age (at the time of tumor resection), NF status, gender, and tumor types, are listed in Table 1 . The diagnosis of NF1 or NF2 in all patients was made using currently 
established clinical diagnostic criteria [1-2]. Two NF1 patients concurrently had a malignant nerve sheath tumor (MPNST) in a different anatomical location. One of the patients with plexiform neurofibroma subsequently developed MPNST in a different location, with a latency of 7 years.

\section{PD-L1 Expression}

PD-L1 expression on tumor cell membrane has been recognized as a predictive marker associated with therapeutic response with anti-PD-1/anti PD-L1 checkpoint inhibitor antibodies [15]. Among the commercially available antibodies, we chose two rabbit monoclonal antibodies that target the cytoplasmic domain of PD-L1. These two antibodies have been reported to be more specific and sensitive compared to other clones [16]. Tumor cells were identified based on morphology and compared with the hematoxylin and eosin stain (H\&E) on corresponding adjacent tissue sections. Positive expression for PD-L1 was defined as membranous staining in greater than $5 \%$ of tumor cells. Positive PD-L1 expression expression using two clones (SP142 and E1L3N, respectively) was observed in $6 / 6(100 \%)$ and 5/6 (83\%) of NF1 plexiform neurofibromas, $8 / 10(80 \%)$ and $6 / 10(60 \%)$ of NF1 dermal neurofibromas, 7/10 (70\%) and 10/10 (100\%) of NF2 schwannomas, and 4/10 $(40 \%)$ and 2/10 (20\%) of NF2 meningiomas. In $68 \%$ of examined tumor tissues, the results were concordant with both antibodies. Taken together, these data indicate that PD-L1 is heterogeneously expressed in NF associated tumors, with the highest percentage of PD-L1 positive tumor cells and strongest PD-L1 expression observed in NF1 plexiform neurofibromas and NF2 schwannomas (Fig. 1).

\section{Tumor-infiltration Lymphocytes and Macrophages}

Tumor response to immune checkpoint inhibitors is not only associated with infiltration of lymphocytes in general, but also associated the type and location of lymphocytic infiltrate [15]. Tumors with baseline infiltration of CD8+ cytotoxic lymphocytes are generally considered good candidates for checkpoint inhibitor therapy. We further characterized the NF tumor immune microenvironment by immunohistochemical staining with macrophage and lymphocyte markers, with CD68 staining for macrophages, CD3 for all mature T cells, CD8 for cytotoxic T cells, and CD20 for B cells (Fig2.). Comparing immunohistochemical and $\mathrm{H} \& \mathrm{E}$ slides, tumor cells did not aberrantly express any of the inflammatory markers. The distribution pattern and cell density of TILs is summarized in Table 2. We found sparse to moderate presence of CD68, CD3, or CD8 positive TILs in 36/36 (100\%) of tumor specimens. However, expression of CD20+ B lymphocytes was limited to rare intratumoral cells detectable in 4/36 (11\%) of tumors. We found CD20+ cells to be predominantly located in proximity to tumor vasculature in NF2 meningiomas and schwannomas (Fig 2, C\&G). The distribution of CD3+ and CD8+ T cells was found to be mostly patchy, while CD68+ macrophages were more diffusely present.

\section{Association of Tissue-Based Markers}

Utilizing immunohistochemistry for PD-L1 (clone SP142) and CD8 performed on adjacent tissue sections, we found a positive association between PD-L1 (clone SP142) positivity and CD8+ TIL density ( $\mathrm{p}$ < 0.03) (Table 3). Although for technical reasons, (i.e. re-cutting of tissue blocks), immunostains for CD8 and PD-L1 clone 1EL3N were not performed on 
immediately adjacent tissue sections, we still found higher levels of CD8+ cell infiltration in PD-L1 positive tumors compared to PD-L1 negative tumors (Table 3). This result provides further evidence that adaptive resistance to cell-mediated immunity plays a major role in the tumor immune microenvironment of NF related tumors.

\section{Conclusions}

PD-L1 expression by tumor cells and CD8 cytotoxic T cell infiltration are well-recognized predictive biomarkers for response to immune checkpoint inhibitors [15]. Previous publications have demonstrated PD-L1 expression in schwannomas [17] and meningiomas [18], but these series included sporadic tumors and did not focus specifically on NF patients. Loss of NF1 in tumors has been shown to lead to macrophage infiltration [19-20], and the subsequent increase in cytokines may facilitate recruitment of CD8+ cytotoxic T cells [21-22]. However, the specific mechanisms leading to TIL recruitment and upregulation of PD-L1 in the context of NF1 and NF2 tumorigenesis merit further investigation.

Our findings indicate that adaptive resistance to cell-mediated immunity plays a major role in the tumor immune microenvironment of NF1 and NF2 associated tumors. The presence of TILs and high expression of PD-L1 on tumor cells suggests that NF patients with plexiform neurofibromas and vestibular schwannomas may be responsive to immunotherapy with immune checkpoint inhibitors, which should be explored in clinical trials.

Recent data indicates that the therapeutic efficacy of checkpoint inhibitors may be augmented by targeted molecular therapy [23]. The combination of BRAF/MAP kinase and immune checkpoint inhibition may increase therapeutic efficacy [24], and VEGF as well as PI3-Akt-mTOR signaling appears to play a role in PD-L1 expression and recruitment of inhibitory immune cells [6, 25-27]. Based on our findings, and given that therapies targeting VEGF, mTOR and MEK have yielded clinical responses in subsets of NF patients [28-30], exploring combinatorial therapies with checkpoint inhibitors appears to be a fruitful area of further pre-clinical and clinical investigation.

\section{Acknowledgments}

This research was funded in part through the NIH/NCI Cancer Center Support Grant P30 CA008748 to Memorial Sloan Kettering Cancer Center. The NYU Experimental Pathology Immunohistochemistry Core Laboratory is supported in part by the Laura and Isaac Perlmutter Cancer Center Support Grant; NIH/NCI P30 CA016087 and the National Institutes of Health S10 Instrumentation Grants; NIH/ORIP S10 OD01058 and S10 OD018338. This study was presented in part at the Biennial 2017 Pediatric Neuro-Oncology Basic and Translational Research Conference, New York, NY, June 2017, and the Children's Tumor Foundation 2017 NF Conference, Washington, DC, June 2017.

\section{References}

1. Gutmann DH, Ferner RE, Listernick RH, Korf BR, Wolters PL, Johnson KJ. Neurofibromatosis type 1. Nat Rev Dis Primers. 2017; 3:17004. 23. doi: 10.1038/nrdp.2017.4 [PubMed: 28230061]

2. Ardern-Holmes S, Fisher G, North K. Neurofibromatosis Type 2. J Child Neurol. 2017; 32(1):9-22. DOI: 10.1177/0883073816666736 [PubMed: 27655473]

3. Karajannis MA, Ferner RE. Neurofibromatosis-related tumors:emerging biology and therapies. Curr Opin Pediatr. 2015; 27(1):26-33. DOI: 10.1097/MOP.0000000000000169 [PubMed: 25490687] 
4. Monroe CL, Dahiya S, Gutmann DH. Dissecting Clinical Heterogeneity in Neurofibromatosis Type 1. Annu Rev Pathol. 2017; 24(12):53-74. DOI: 10.1146/annurev-pathol-052016-100228

5. Chamoto K, Al-Habsi M, Honjo T. Role of PD-1 in Immunity and Diseases. Curr Top Microbiol Immunol. 2017; 410:75-97. DOI: 10.1007/82_2017_67 [PubMed: 28929192]

6. Kythreotou A, Siddique A, Mauri FA, Bower M, Pinato DJ. PD-L1. J Clin Pathol. 2017; doi: 10.1136/jclinpath-2017-204853

7. Chen J, Jiang CC, Jin L, Zhang XD. Regulation of PD-L1: a novel role of pro-survival signalling in cancer. Ann Oncol. 2016; 27(3):409-16. DOI: 10.1093/annonc/mdv615 [PubMed: 26681673]

8. Wang X, Huang S, Zhang Y, Zhu L, Wu X. The application and mechanism of PD pathway blockade for cancer therapy. Postgrad Med J. 2017; doi: 10.1136/postgradmedj-2017-135187

9. Balar AV, Weber JS. PD-1 and PD-L1 antibodies in cancer: current status and future directions. Cancer Immunol Immunother. 2017; 66(5):551-564. DOI: 10.1007/s00262-017-1954-6 [PubMed: 28213726]

10. Fujiwara N, Kobayashi K. Macrophages in inflammation. Curr Drug Targets Inflamm Allergy. 2005; 4(3):281-6. [PubMed: 16101534]

11. Prada CE, Jousma E, Rizvi TA, Wu J, Dunn RS, Mayes DA, Cancelas JA, Dombi E, Kim MO, West BL, Bollag G, Ratner N. Neurofibroma-associated macrophages play roles in tumor growth and response to pharmacological inhibition. Acta Neuropathol. 2013; 125(1):159-68. DOI: 10.1007/s00401-012-1056-7 [PubMed: 23099891]

12. Shi Y, Bollam SR, White SM, Laughlin SZ, Graham GT, Wadhwa M, Chen H, Nguyen C, Vitte J, Giovannini M, Toretsky J, Yi C. Rac1-Mediated DNA Damage and Inflammation Promote Nf2Tumorigenesis but Also Limit Cell-Cycle Progression. Dev Cell. 2016; 39(4):452-465. DOI: 10.1016/j.devcel.2016.09.027 [PubMed: 27818180]

13. Park SJ, Sawitzki B, Kluwe L, Mautner VF, Holtkamp N, Kurtz A. Serum biomarkers forneurofibromatosis type 1 and early detection of malignant peripheral nerve-sheath tumors. BMC Med. 2013; 23(11):109.doi: 10.1186/1741-7015-11-109

14. Li H, Velasco-Miguel S, Vass WC, Parada LF, DeClue JE. Epidermal growth factor receptor signaling pathways are associated with tumorigenesis in the Nf1:p53 mouse tumor model. Cancer Res. 2002; 62(15):4507-13. [PubMed: 12154062]

15. Dong ZY, Wu SP, Liao RQ, Huang SM, Wu YL. Potential biomarker for checkpoint blockade immunotherapy and treatment strategy. Tumour Biol. 2016; 37(4):4251-61. DOI: 10.1007/ s13277-016-4812-9 [PubMed: 26779629]

16. Mahoney KM, Sun H, Liao X, Hua P, Callea M, Greenfield EA, Hodi FS, Sharpe AH, Signoretti S, Rodig SJ, Freeman GJ. PD-L1 Antibodies to Its Cytoplasmic Domain Most Clearly Delineate Cell Membranes in Immunohistochemical Staining of Tumor Cells. Cancer Immunol Res. 2015; 3(12): 1308-15. DOI: 10.1158/2326-6066.CIR-15-0116 [PubMed: 26546452]

17. Archibald DJ, Neff BA, Voss SG, Splinter PL, Driscoll CL, Link MJ, Dong H, Kwon ED. B7-H1 expression in vestibular schwannomas. Otol Neurotol. 2010; 31(6):991-7. DOI: 10.1097/MAO. 0b013e3181e40e4f [PubMed: 20601920]

18. Han SJ, Reis G, Kohanbash G, Shrivastav S, Magill ST, Molinaro AM, McDermott MW, Theodosopoulos PV, Aghi MK, Berger MS, Butowski NA, Barani I, Phillips JJ, Perry A, Okada H. Expression and prognostic impact of immune modulatory molecule PD-L1 in meningioma. $\mathbf{J}$ Neurooncol. 2016; 130(3):543-552. [PubMed: 27624915]

19. Prada CE, Jousma E, Rizvi TA, Wu J, Dunn RS, Mayes DA, Cancelas JA, Dombi E, Kim MO, West BL, Bollag G, Ratner N. Neurofibroma-associated macrophages play roles in tumor growth and response to pharmacological inhibition. Acta Neuropathol. 2013; 125(1):159-68. DOI: 10.1007/s00401-012-1056-7 [PubMed: 23099891]

20. Yang FC, Ingram DA, Chen S, Zhu Y, Yuan J, Li X, Yang X, Knowles S, Horn W, Li Y, Zhang S, Yang Y, Vakili ST, Yu M, Burns D, Robertson K, Hutchins G, Parada LF, Clapp DW. Nf1dependent tumors require a microenvironment containing Nf1+/-- and c-kit-dependent bone marrow. Cell. 2008; 31135(3):437-48. DOI: 10.1016/j.cell.2008.08.041

21. Solga AC, Pong WW, Kim KY, Cimino PJ, Toonen JA, Walker J, Wylie T, Magrini V, Griffith M, Griffith OL, Ly A, Ellisman MH, Mardis ER, Gutmann DH. RNA Sequencing of TumorAssociated Microglia Reveals Ccl5 as a Stromal Chemokine Critical for Neurofibromatosis-1 
Glioma Growth. Neoplasia. 2015; 17(10):776-88. DOI: 10.1016/j.neo.2015.10.002 [PubMed: 26585233]

22. Zhao, Li, Jianglin, Xu, Xiaobing, Liu, Rongbin, Guo, Pengfei, Wang. Dysregulation of IL-10 by macrophages and T cells in recurrent and malignant schwannomas. Int J Clin Exp Pathol. 2016; 9:7302-7309.

23. Ingram DA, Zhang L, McCarthy J, Wenning MJ, Fisher L, Yang FC, Clapp DW, Kapur R. Lymphoproliferative defects in mice lacking the expression of neurofibromin:functional and biochemical consequences of Nf1 deficiency in T-cell development and function. Blood. 2002; 100(10):3656-62. [PubMed: 12393709]

24. Liu L, Mayes PA, Eastman S, Shi H, Yadavilli S, Zhang T, Yang J, Seestaller-Wehr L, Zhang SY, Hopson C, Tsvetkov L, Jing J, Zhang S, Smothers J, Hoos A. The BRAF and MEK Inhibitors Dabrafenib and Trametinib: Effects on Immune Function and in Combination with Immunomodulatory Antibodies Targeting PD-1,PD-L1, and CTLA-4. Clin Cancer Res. 2015; 121(7):1639-51. DOI: 10.1158/1078-0432.CCR-14-2339

25. Wada J, Suzuki H, Fuchino R, Yamasaki A, Nagai S, Yanai K, Koga K, Nakamura M, Tanaka M, Morisaki T, Katano M. The contribution of vascular endothelial growth factor to the induction of regulatory T-cells in malignant effusions. Anticancer Res. 2009 Mar; 29(3):881-8. [PubMed: 19414323]

26. Osada T, Chong G, Tansik R, Hong T, Spector N, Kumar R, Hurwitz HI, Dev I, Nixon AB, Lyerly HK, Clay T, Morse MA. The effect of anti-VEGF therapy on immature myeloid cell and dendritic cells in cancer patients. Cancer Immunol. 2008

27. Parsa AT, Waldron JS, Panner A, Crane CA, Parney IF, Barry JJ, Cachola KE, Murray JC, Tihan T, Jensen MC, Mischel PS, Stokoe D, Pieper RO. Loss of tumor suppressor PTEN function increases B7-H1 expression and immunoresistance in glioma. Nat Med. 2007; 13(1):84-8. [PubMed: 17159987]

28. Goutagny S, Kalamarides M. Medical treatment in neurofibromatosis type 2. Review of the literature and presentation of clinical reports. Neurochirurgie. 2017; 2doi: 10.1016/j.neuchi. 2016.09.004

29. Blakeley JO, Ye X, Duda DG, Halpin CF, Bergner AL, Muzikansky A, Merker VL, Gerstner ER, Fayad LM, Ahlawat S, Jacobs MA, Jain RK, Zalewski C, Dombi E, Widemann BC, Plotkin SR. Efficacy and Biomarker Study of Bevacizumab for Hearing Loss Resulting From Neurofibromatosis Type 2-Associated Vestibular Schwannomas. J Clin Oncol. 2016; 34(14):166975. DOI: 10.1200/JCO.2015.64.3817 [PubMed: 26976425]

30. Dombi E, Baldwin A, Marcus LJ, Fisher MJ, Weiss B, Kim A, Whitcomb P, Martin S, AschbacherSmith LE, Rizvi TA, Wu J, Ershler R, Wolters P, Therrien J, Glod J, Belasco JB, Schorry E, Brofferio A, Starosta AJ, Gillespie A, Doyle AL, Ratner N, Widemann BC. Activity of Selumetinib in Neurofibromatosis Type 1-Related Plexiform Neurofibromas. N Engl J Med. 2016; 375(26):2550-2560. DOI: 10.1056/NEJMoa1605943 [PubMed: 28029918] 

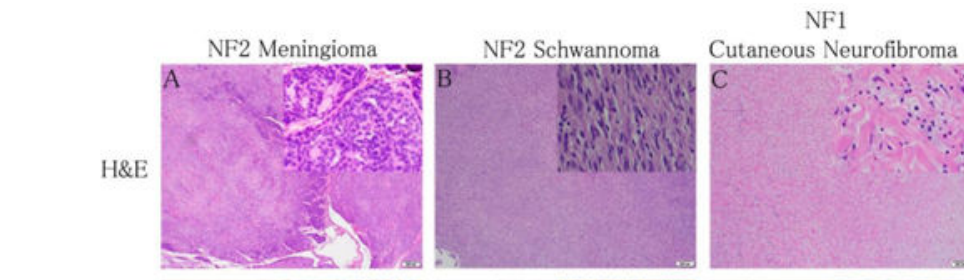

Plexiform Neurofibroma
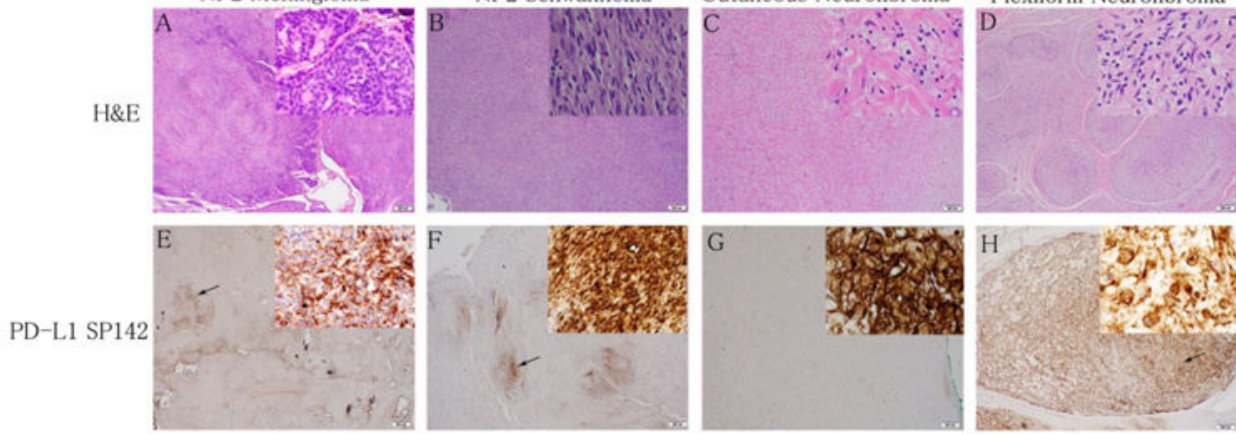

PD-L1 E1L3N
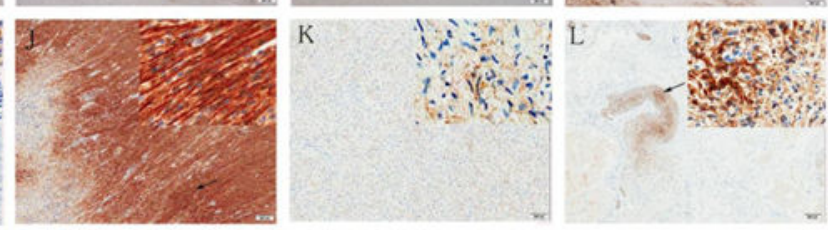

Figure 1. Immunohistochemistry staining of PD-L1 expression in NF associated tumors This figure illustrates the PD-L1 expression at low magnification (100x) and high magnification (400×) with monoclonal antibodies, clone SP142 (E to H) vs. E1L3N (I to L) in NF2 meningioma (E vs. I), NF2 schwannoma (F vs. J), NF1 cutaneous neurofibroma (G vs. K), and NF1 plexiform neurofibroma (H vs. L). H\&E stains (A to D) indicate the morphology of NF related tumors: NF2 meningioma (A), NF2 schwannoma (B), NF1 cutaneous neurofibroma (C), and NF1 plexiform neurofibroma (D) at low magnification $(100 \times)$ and high magnification $(400 \times)$. 


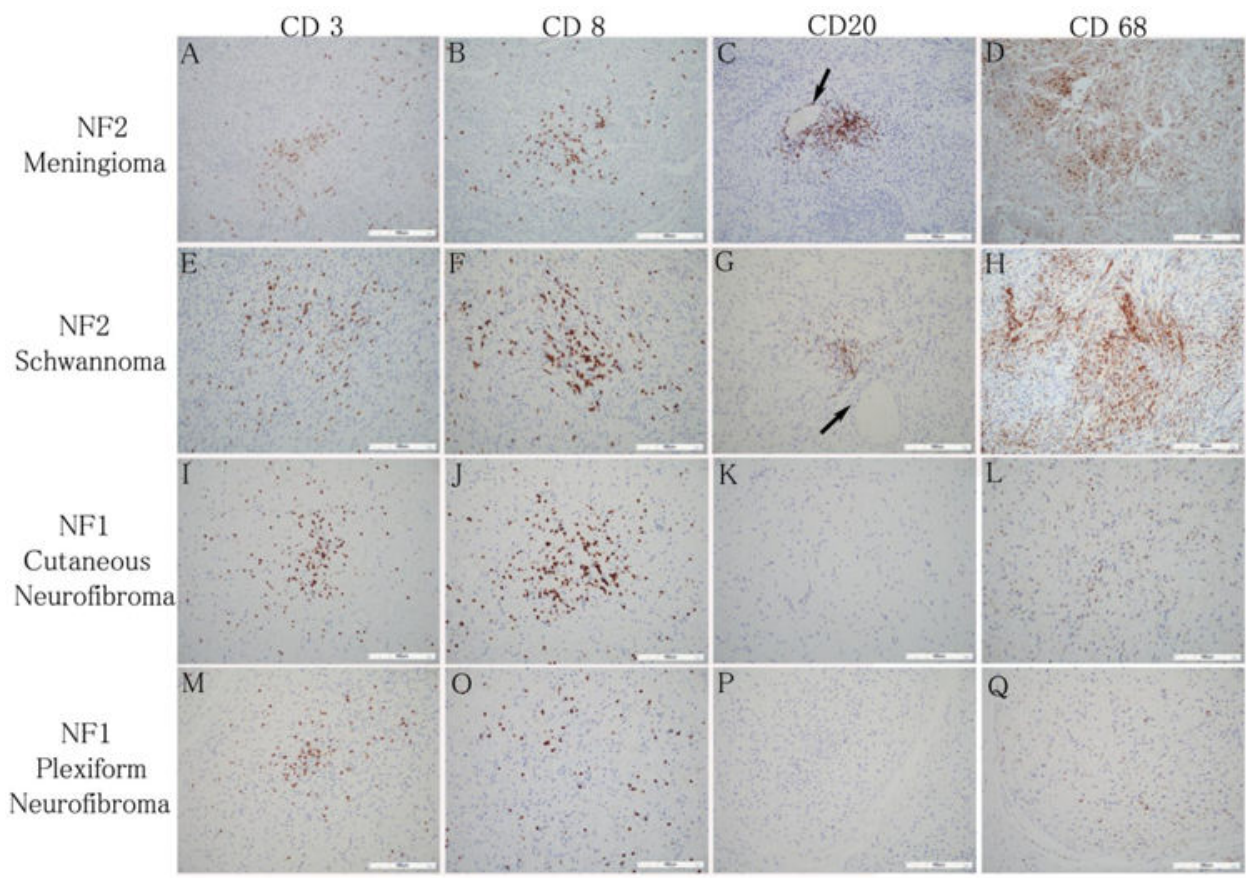

Figure 2. Tumor-infiltrating lymphocytes and macrophages in NF-related tumors Immunohistochemistry stain of CD3, CD8, CD68, and CD20 positive lymphocytes and macrophages in NF2 meningioma (A-D), NF2 schwannoma (E-H), NF1cutanous neurofibroma (I-L), and NF1 plexiform neurofibroma (M-P). This figure reveals the infiltration of $\mathrm{CD} 3+(\mathrm{A}, \mathrm{E}, \mathrm{I}$, and $\mathrm{M})$ and $\mathrm{CD} 8+(\mathrm{B}, \mathrm{F}, \mathrm{J}$, and $\mathrm{N}) \mathrm{T}$ lymphocytes and CD68+ (D, H, L, and P) macrophages in tumor tissues, not CD20+ B cells (C, G, K, and O). "Leaking" of CD20+ B cells in meningioma and schwannoma is shown in (C and G), with arrows indicating the vascular structures. 


\section{Table 1}

\section{Summary of Patient Characteristics}

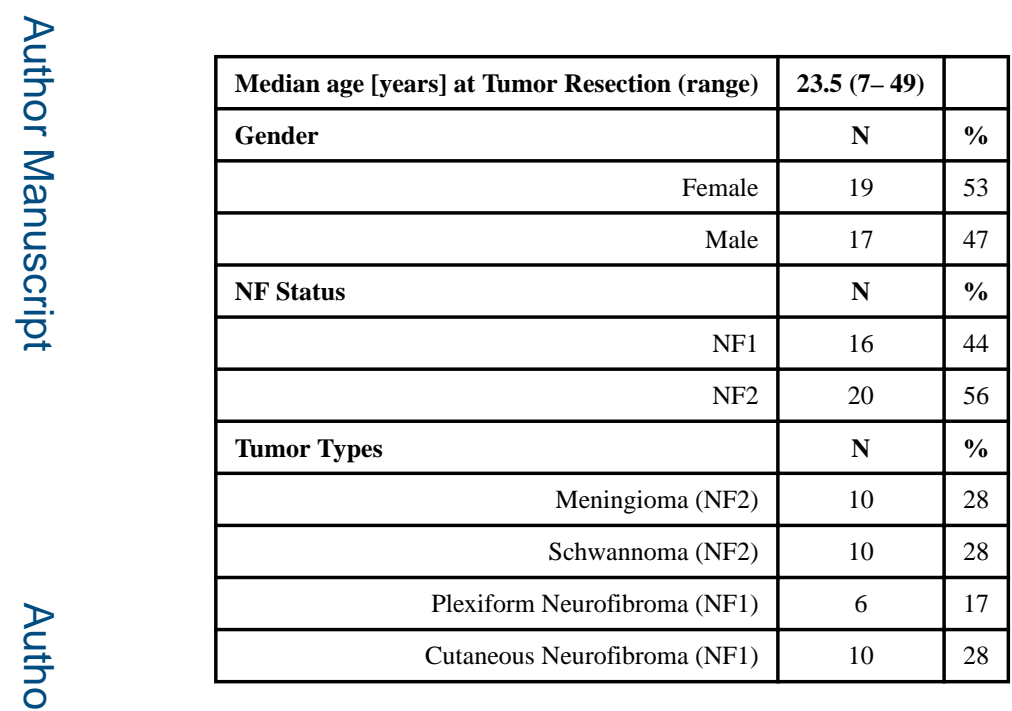




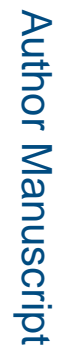

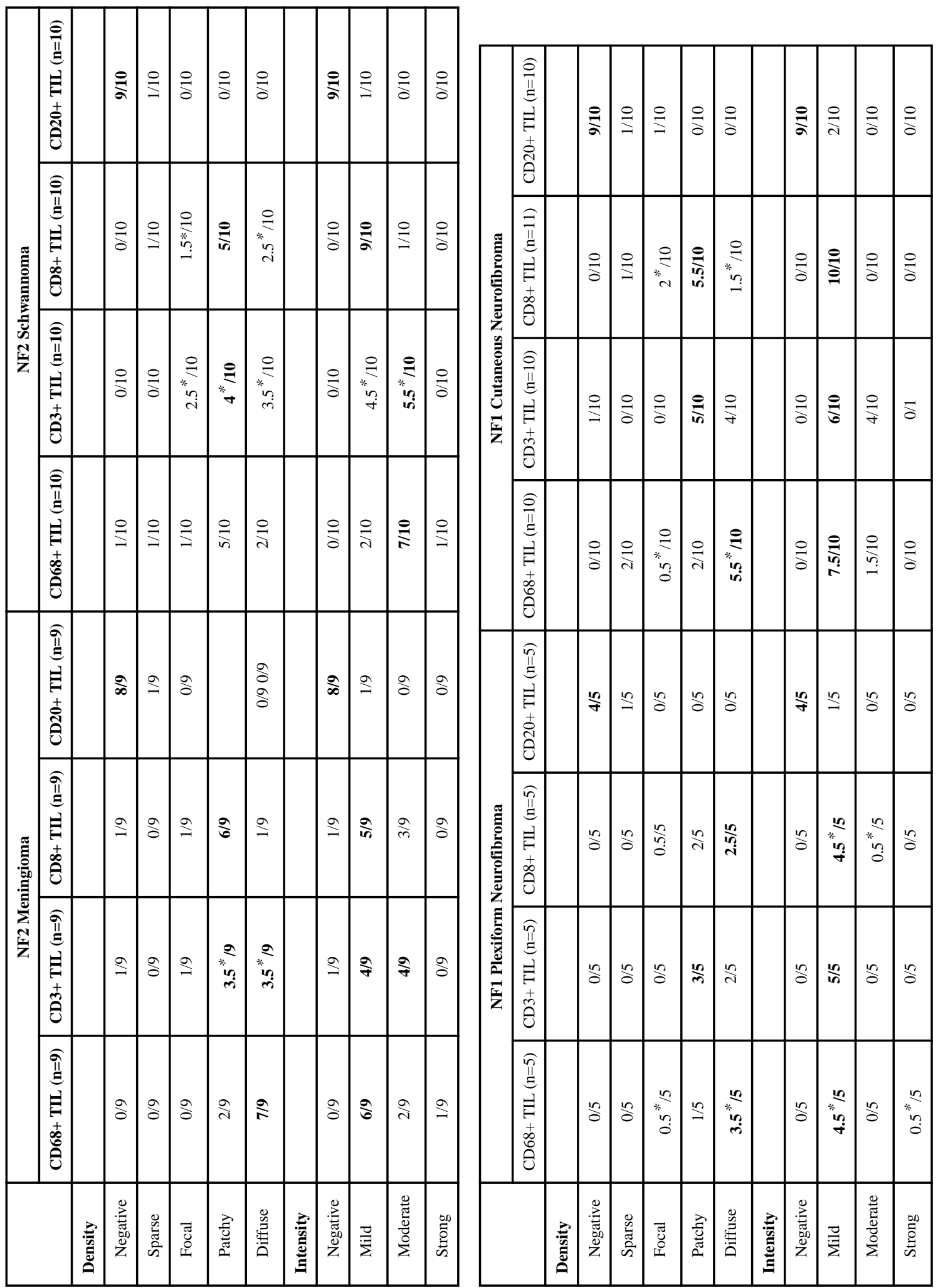

J Neurooncol. Author manuscript; available in PMC 2019 May 01. 

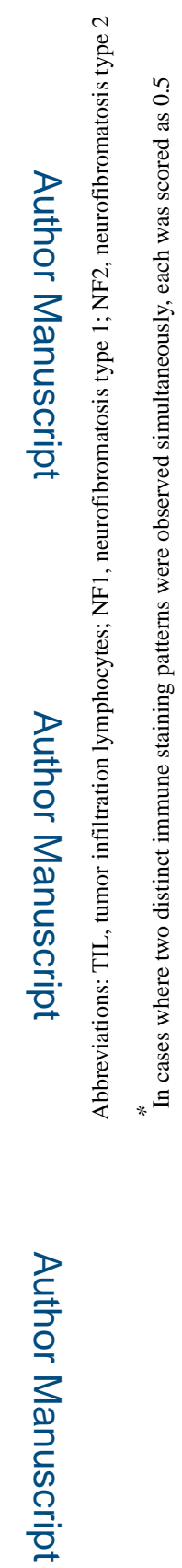

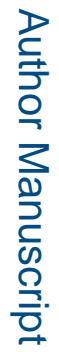

J Neurooncol. Author manuscript; available in PMC 2019 May 01. 
Table 3

Summary of PD-L1 expression and CD8 cytotoxic T cell tumor infiltration (sp142 vs.

1EL3N)

\begin{tabular}{|c|c|c|c|c|}
\hline \multirow{2}{*}{ Number of CD8 positive T cells } & \multicolumn{2}{|c|}{ PD-L1 (sp142) } & \multicolumn{2}{|c|}{ PD-L1 (1EL3N) } \\
\hline & Positive (n) & Negative (n) & Positive (n) & Negative (n) \\
\hline $0<=25$ cells $/ 400 \times$ & 3 & 6 & 6 & 2 \\
\hline$<25<=50$ cells $/ 400 \times$ & 5 & 3 & 4 & 4 \\
\hline$<50<=100$ cells $/ 400 \times$ & 12 & 1 & 11 & 1 \\
\hline$>100$ cells $/ 400 x$ & 3 & 1 & 2 & 2 \\
\hline Total: & 23 & 11 & 23 & 11 \\
\hline
\end{tabular}

For sp142: The chi-square statistic is 8.6499 . The $\mathrm{p}$-value is 0.03 (significant at $p<0.05$.

For 1EL3N: The chi-square statistic is 5.2045. The p-value is 0.17 (not significant at $p<0.05$ ) 